\title{
Consideration for Employer-Based and Geographic Attributes Included in Value Assessment Methods of Next-Generation Sequencing Tests
}

\author{
M. Ragan Hart, MS, PhD, and Scott J. Spencer, MPA, MA
}

\begin{abstract}
SUMMARY
There is a need for formal cost-effectiveness evidence to better model the real-world payer decision context in which general economic models are currently being used, specifically regarding clinical genomics health services (for next-generation sequencing (NGS) tests). We reviewed literature focused on cost-effectiveness studies after completion of the Human Genome Project within the Tufts Cost-Effectiveness Analysis (CEA) Registry and found that only $33 \%$ of eligible studies were conducted from the U.S. payer perspective. Additional interpretation challenges include economic models that do not account for true payer-negotiated costs, limits in internal expertise for quality-adjusted life-year inferences, and limited internal policies to use CEA research in decision making. This Viewpoints article highlights numerous opportunities to increase the translational effect of economic modeling work. Specifically, geographically relevant cost and outcomes data should be considered for integration within best practices for economic evaluations of NGS tests. Such data integration may provide more informed decision making regarding the allocation of constrained resources for health care services and technology.
\end{abstract}

J Manag Care Spec Pharm. 2019;25(8):936-40

Copyright $\odot 2019$, Academy of Managed Care Pharmacy. All rights reserved.

A $\mathrm{s}$ previously described in the literature, there is a need for formal cost-effectiveness evidence to better model the real-world payer decision context in which general economic models are currently being used for health technology assessment. ${ }^{1,2}$ This need is especially important to consider with regard to clinical genomics health services and complementary to the broader discourse regarding forthcoming perspectives on value. ${ }^{3}$

\section{Next-Generation Sequencing Tests}

Clinical genomics market entrants continue to increase, with a recent analysis reporting nearly 75,000 tests currently available and nearly 10 new tests added daily. ${ }^{4}$ Consequently, health technology assessment groups and health care decision makers must review available evidence to discern clinical and economic utility of genomics-based tests to inform medical policy and decision making regarding coverage and/or reimbursement decisions. One comprehensive, open access tool has been developed to support health care decision makers in their value assessment process for companion diagnostic tests (a type of next-generation sequencing [NGS] $).{ }^{5}$ However, it is unknown how often this tool is used. Further, its use may be limited for stand-alone NGS tests that are not informing a drug treatment decision, but rather the NGS test results inform different medical management. Empirical data show that the majority of U.S. spending by quarter from 2014 to 2016 was on multigene panels, yet the health economics evidence base is lacking comprehensive value assessments of test options. ${ }^{4}$

We denote genomics-based tests hereafter as NGS tests, although different methods can be used (e.g., array-based genotyping). Given the availability of over 75,000 NGS tests, the current state of U.S. payer decision making for coverage of NGS tests varies across self-insured employers, commercial, and public payer programs. To better understand the state of the field and market options, we reviewed a subset of economic evaluations, identified shortcomings in currently available economic evidence, and proposed solutions.

\section{Economic Evaluations After Completion} of Human Genome Project

We reviewed available literature from the Tufts CostEffectiveness Analysis (CEA) Registry, ${ }^{6}$ including studies that reported on cost-effectiveness, after completion of the Human Genome Project, which publicly released its complete data in 2003. Following our review of economic analyses characterized in the CEA Registry (nonsponsor access), there were 46 studies that met our criteria of cost analysis after completion of the genome project. Of those evaluations, one third (15/46) of cost-effectiveness studies evaluating NGS tests (notably, genotyping array comparators predominated as the intervention under assessment) conducted to date used the U.S. payer perspective model parameter assumptions under the guise of a type of payer. The additional evaluations undertook either a societal perspective, a non-U.S. payer, or the perspective could not be determined (Figure 1).

For the 15 studies conducted from the U.S. payer perspective, there was variation in the time horizons evaluated (range: 1 year to lifetime, with 8 of the studies modeling a lifetime horizon). From a payer perspective assuming employer-sponsored insurance, 3 years is often an appropriate time horizon, due to the effect of employee retention, and evidence from the studies reviewed here with a longer time frame may be less informative to specific payer decision makers. One analysis aptly described that "[their] model estimates total costs for the U.S. health care system as a whole rather than expenditures incurred by specific payers." Given that a national health care system does not exist in the United States, evidence from investigations using such a methodology have limited inferences 


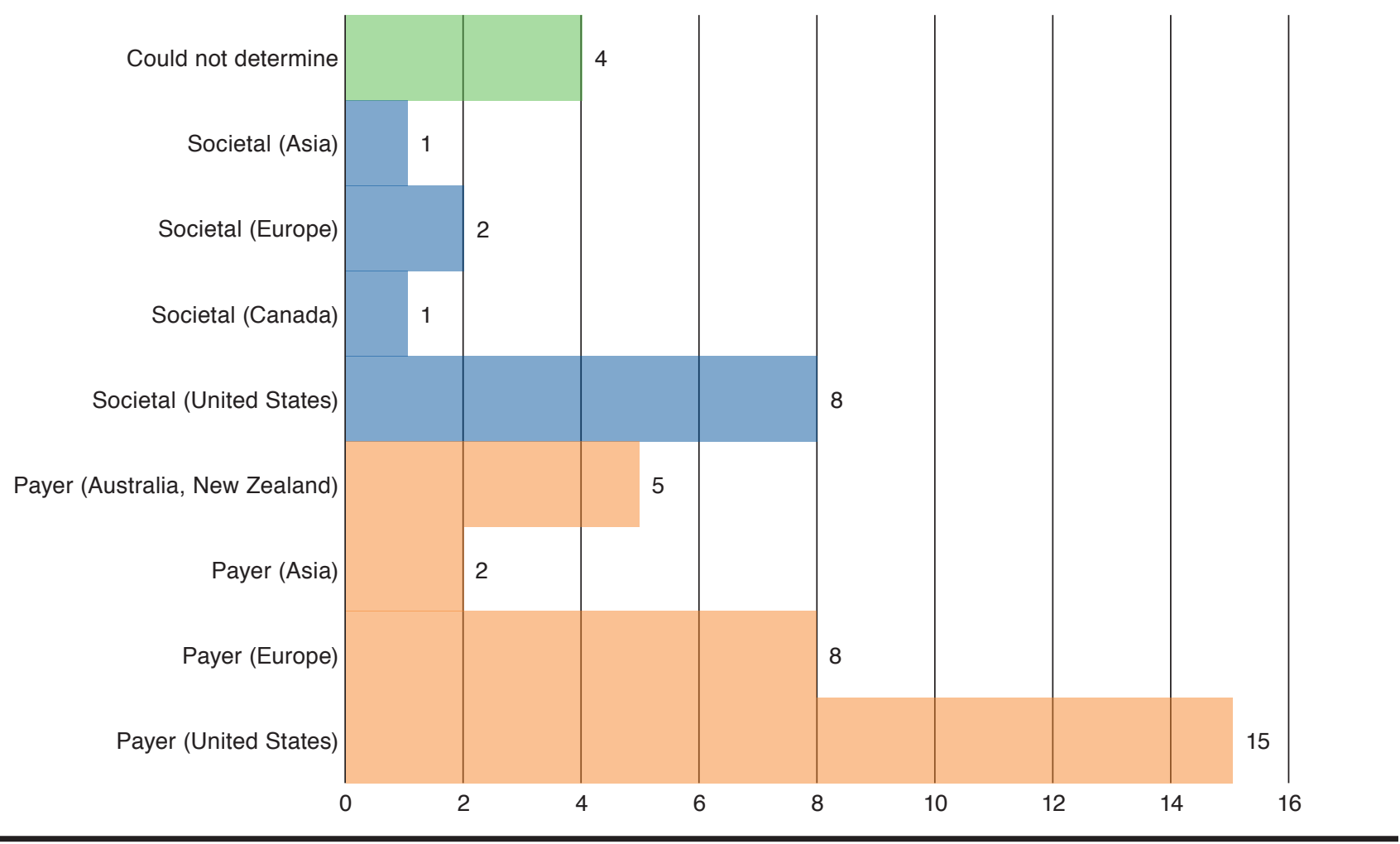

to the various types of payer or industry stakeholders determining coverage for NGS tests without the inclusion of more precise geographically relevant parameters or factors. Notably, however, the argument posited here may provide informative recommendations for public or other types of private payers, since they may consider the variation in geographic dynamics that exist within their respected covered populations.

\section{Using Geographic-Specific Data for Self-Insured Employers}

Acknowledging that there are multiple payer types and health plan structures, we focus on self-insured (or self-funded) employers determining appropriate health services on behalf of their employee beneficiaries as the entity responsible for covering health expenses directly. Employers are tasked with developing and improving strategies for population health management. ${ }^{8}$ While we focus on the proposed solutions for self-insured employers hereafter described, incorporating geographic variability data into other payer perspectives (e.g., public and private) may also be relevant to policy coverage regarding NGS testing. Applying payer-specific model inputs with an increased focus on integrating self-insured employer parameter preferences may render health economic models more useful in a real-world decision-making context.
The U.S. Census 2014 population estimate was 319 million, and the Kaiser Family Foundation reported that 49\% (estimated to be 156 million individuals) of the U.S. population were covered by employer-sponsored insurance. ${ }^{9}$ This estimate excludes those receiving insurance under Medicaid, Medicare, nongroup, other public, and those uninsured. Using data from a recent Department of Labor report to Congress, self-insured employer plans covered an estimated 33 million participants in 2014, which comprised approximately one fifth (21\%) of the estimated covered lives within the U.S. population. ${ }^{10}$ This commentary highlights and elucidates challenges and implications regarding current methods for generating economic evidence for NGS tests to inform value assessments by health care decision makers, using self-insured employer plans as a case to highlight.

Although beyond the scope of this commentary, it is worth noting that the "payer" perspective often may be unclear in CEA work conducted, since such analyses modestly report on size, scope of payer decision context, and affiliation of the payer used for analysis. While we realize that this may be because of data limitations, unwillingness for collaboration, or various other reasons, this does not diminish that more could be done to provide increased transparency and clarity around 
data sources from specific payer perspectives. In addition, from our list of CEAs evaluated, rarely is a comparison of public versus private reimbursement rate reported in the cost assumptions and/or limitations, precluding potentially informative empirical cost data.

\section{Current Limitations of Value Assessment} Evidence for NGS Tests

Health care decision makers (insurers, self-insured employers, and third-party administrators) cite limitations in published health economic models. ${ }^{1}$ These models include those not accounting for the true payer-negotiated cost of the intervention, limits in internal expertise to discern quality-adjusted life-year (QALY) inferences, and no formal internal policies to use CEA research in decision making. Several economic analyses report on cost-effective strategies using genetic testing ${ }^{11,12}$; however, understanding the willingness-to-pay thresholds employed are limited with no comparison of effect regarding resource allocation for other health services. Although, in 1 CEA, the authors acknowledged their decision to use a shorter time horizon, noting that the results may be easier to interpret from a payer perspective and would not be as affected by discount rates (as would be results modeled under longer time horizons). ${ }^{12}$ They even modeled cost offsets to support the interpretation of their results, which is not always reported in the literature.

Additional challenges present when formally evaluating the economic consequences of new interventions. This perspective intends to extend the discussion of several methodological issues regarding assessment of economic value of NGS tests. ${ }^{13}$ Phillips et al. (2018) identified complex model structure and data availability and quality issues as challenges prevalent within the value assessment process. ${ }^{13}$ Further, this Viewpoints article aims to address several points associated with reimbursement challenges that have previously been identified as barriers to genetic testing. ${ }^{14}$

\section{Proposed Solutions for Improving Current Methods}

Using a calculation that can cross-walk/convert an incremental cost-effectiveness ratio for a specific willingness-to-pay threshold into the effect on a per-member-per-month (PMPM), or even annual member, estimate may increase applicability of available economic evidence around NGS tests. While budget impact modeling (BIM) methods provide some insight into the PMPM estimate (an estimate that payers more readily favor and may be comfortable interpreting), such models may be less robust to the best practice methods used for CEAs. Both methods are intended to be used as complements to inform spending priorities (CEAs) and estimate new expenditures along with projected cost offsets. ${ }^{15}$ However, developing a hybrid approach with features of both methods may be better able to capture the value associated with NGS tests. Genetic information generated may provide perpetual value during more than a single clinical decision. Instead, it may be necessary to model value associated with multiple decision points within a clinical scenario, which is a perturbation from current modeling convention.

For example, one solution may be by bridging selected parameters for a CEA and incorporating the same data elements into a BIM. Assuming the same input estimates across all self-insured employer types presents flaws in real-world applicability. Notably, a recent study found large variability in health care resource utilization and costs across geographic regions ${ }^{16}$ and if conventional CEA models do not account for some of this variation in the modeling parameter estimates, the inference of the models remains limited. Alternatively, while requiring further validation, a different methodological approach has been developed to model an integration of CEA and BIA by a step-wise comparison of the selected thresholds first for willingness-to-pay and next by maximum available budget. ${ }^{17}$

Although our focus excludes health plan benefits covered by policy coverage decisions authorized by the Centers for Medicare \& Medicaid Services (CMS), we do offer brief credence to implications that CMS may have on commercial plans. The Medicare administrative contractors serve a specific geographical area or jurisdiction and follow the Molecular Diagnostic Services (MolDx) coverage decisions that confirm that a NGS test meets Medicare reasonable and necessary criteria, ${ }^{18}$ and using a model relevant to self-insured employers may be extensible to MolDx's technical assessment and evidence review process. In addition, the Concert Genetics resource, which aggregates laboratory, health claims, and health plan data (query functions available via a free account) reports variation in costs for the same NGS test across multiple laboratories nationally and inconsistencies for what payers are actually paying. ${ }^{19}$

Such variation engenders information asymmetry because of a lack of transparency and disallows true cost data to be appropriately included as more realistic model inputs. Including cost variation data that may mitigate information asymmetry could improve cost management strategies. This improvement may occur by more accurate modeling of per capita spending and health gain effects and providing evidence that informs the projected cost estimates that are more representative of the geographic area of focus in which a self-insured employer operates.

For example, the cost inputs of the same PCSK9 genetic test offered by 2 geographically distinct laboratories are $\$ 890$ (California) and \$1,600 (Ohio), respectively, because of market rate variations and differences in analysis approach for the same gene. ${ }^{19}$ The local prevalence of heterozygous familial hypercholesterolemia (for which the aforementioned test is used) varies from 1 in 200 to 1 in 500 (California) to greater than 1 in 165 (areas within Ohio). ${ }^{20,21}$ Therefore, modeling a 


\section{Consideration for Employer-Based and Geographic Attributes Included in Value Assessment Methods of Next-Generation Sequencing Tests}

fixed cost and prevalence within an economic evaluation of a PCSK9 genetic test and associated targeted drug therapies limits relevance for decision makers across multiple regions. While sensitivity analyses are intended to account for some of this variation, specifying bounded estimates to reflect regional market data may be more practically informative. In addition, some self-insured employers may be accountable for population health management beyond the previously mentioned 3-year time horizon. Accordingly, modeling the cost-benefit impacts across a longer time horizon is of potential interest and can result in different evidentiary threshold determinations of a genetic test being cost-effective.

By ascribing to the good reporting practices checklist of CHEERS,$^{22}$ we suggest an additional approach to more robustly characterize uncertainty. This suggestion is timely, given that the CHEERS checklist is up for a review by the authors for necessary updates. We propose that scenario analyses ought to be conducted from regional perspectives, when available, to increase the translational impact on decision makers undertaking the payer perspective. Such an approach could offer applied modeling with an integration of more appropriate real-world estimates to bridge theoretical models that do not maximize translational impact. This solution intends to support more transparent information synthesis relevant for decision analysis and evidence review by providing a more accurate estimate of cost impacts and incremental health outcomes. Furthermore, implementing the approaches previously described may better support a shift in understanding the evidence based on mitigating expected losses compared with erring on the side of expected gains (from the test referred to in the section Economic Evaluations After Completion of Human Genome Project of this article). The expected value from this approach reduces burden of loss (risk uncertainty) by providing a more accurate characterization of local clinical outcome risk based on the implementation of regional data sources compared with mixed or population-based data sources.

Moreover, challenges persist for health economists ascertaining employer cost estimates, since such data often are proprietary and may have unintended consequences as a result of informing non-health care coverage decision making (i.e., investor relations decisions). To address these data sharing challenges, a potential solution is to identify overlapping incentives for the employer and the investigator generating evidence to inform a real cost projection for NGS tests under evaluation and to identify the expected trade-offs as defined by all parties.

In addition, a safe harbor environment could be established to host this information exchange. Without accounting for these types of real cost data, such thought exercises result in limited applicability for real-world decision making. Further, continuing to push health economists to highlight these realworld data limitations, cost or otherwise, may potentially add value for employers by facilitating collaboration, investment, and adaptation of the economic modeling work being done today. We assert that by increasing communication with industry stakeholders, the academic community may create innovative opportunities for collaboration and highlight a desire for future data or clinical insights that are not currently available.

\section{Conclusions}

The purpose of this commentary was to emphasize renewed discussion for identifying additional challenges and proposing new solutions to address limitations in current methods available to assess NGS tests. Opportunities to increase the translational impact of modeling work conducted are abundant.

A limitation of this work is that CEAs not indexed within the Tufts CEA Registry were not evaluated. This limitation biases the results by not including evaluations published in nonEnglish journals (the registry is inclusive of studies published in English). In addition, our review of the literature did not account for studies evaluating health benefits beyond QALYs, such as health resource utilization or disability-adjusted lifeyears, which future reviews should include.

Geographically relevant cost and outcomes data should be better integrated within best practices for economic evaluations of NGS tests. Paramount to our work as academicians in this field is to generate objective and relevant economic evidence. Such evidence should allow for more informed decision making regarding the allocation of constrained resources for available health care services and technology under assessment as it relates to coverage decisions.

Since our proposed solutions are not current practice, we did not remark on operationalizing each; they may, in fact, have higher resource costs, either financial, time, labor, or otherwise, to establish. It will be necessary to build consensus to operationalize and implement these proposed solutions within practice to improve current U.S. payer decision-making processes for the evaluation of NGS tests.

\section{Authors}

M. RAGAN HART, MS, PhD, Department of Biomedical Data Science, Stanford University, Stanford, California, and SCOTT J. SPENCER, MPA, MA, Institute for Public Health Genetics, University of Washington, Seattle.

AUTHOR CORRESPONDENCE: M. Ragan Hart, MS, PhD, Department of Biomedical Data Science, Stanford University, 1265 Welch Rd., Stanford, CA 94305. E-mail:hartmr@stanford.edu.

\section{DISCLOSURES}

No outside funding supported the writing of this article. Hart is supported by an unrestricted gift from Pfizer, which played no role in the study referred to in this article. The authors have no conflicts of interest to report. 


\section{Consideration for Employer-Based and Geographic Attributes Included in Value Assessment Methods of Next-Generation Sequencing Tests}

\section{REFERENCES}

1. Solow B, Pezalla EJ. ISPOR's initiative on US value assessment frameworks: the use of cost-effectiveness research in decision making among U.S. insurers. Value Health. 2018;21(2):166-68.

2. Watkins J. Payer perspectives on health technology assessment. J Manag Care Pharm. 2012;18(3):265-67. Available at: https://www.jmcp.org/ doi/10.18553/jmcp.2012.18.3.265

3. Happe LE. Announcing a new article series: perspectives on value [letter]. J Manag Care Spec Pharm. 2019;25(1):8-9.

4. Phillips KA, Deverka PA, Hooker GW, Douglas MP. Genetic test availability and spending: where are we now? where are we going? Health Aff (Millwood). 2018;37(5):710-16.

5. Canestaro WJ, Pritchard DE, Garrison LP, Dubois R, Veenstra DL. Improving the efficiency and quality of the value assessment process for companion diagnostic tests: the Companion test Assessment Tool (CAT) J Manag Care Spec Pharm. 2015;21(8):700-12. Available at: https://www.jmcp. org/doi/10.18553/jmcp.2015.21.8.700

6. Tufts Medical Center. Center for the Evaluation of Value and Risk in Health. CEA Registry. Available at: https://cevr.tuftsmedicalcenter.org/databases/cea-registry. Accessed June 1, 2019.

7. Mvundura M, Grosse SD, Hampel H, Palomaki GE. The cost-effectiveness of genetic testing strategies for Lynch syndrome among newly diagnosed patients with colorectal cancer. Genet Med. 2010;12(2):93-104

8. Frost A, Barrette E, Kennedy K, Brennan N. Health care spending under employer-sponsored insurance: a 10-year retrospective. Health Aff (Millwood). 2018;37(10):1623-31.

9. Henry J. Kaiser Family Foundation. Health insurance coverage of the total population: 2014. Retrieved October 4, 2018. Available at: https://www.kff. org/other/state-indicator/total-population/?currentTimeframe=0\&sortModel= $\% 7 \mathrm{~B} \% 22$ colld $\% 22: \% 22$ Location\%22,\%22sort $\% 22: \% 22$ asc\%22\%7D. Accessed June 1, 2019

10. U.S. Department of Labor. Report to Congress: annual report on selfinsured group health plans. March 2017. Available at: https://www.dol.gov/ sites/default/files/ebsa/researchers/statistics/retirement-bulletins/annualreport-on-self-insured-group-health-plans-2017.pdf. Accessed June 1, 2019.
11. Okere AN, Ezendu K, Berthe A, Diaby V. An evaluation of the cost-effectiveness of comprehensive MTM integrated with point-of-care phenotypic and genetic testing for U.S. elderly patients after percutaneous coronary intervention. J Manag Care Spec Pharm. 2018;24(2):142-52. Available at: https://www.jmcp.org/doi/10.18553/jmcp.2018.24.2.142.

12. Groessl EJ, Tally SR, Hillery N, Maciel A, Garces JA. Cost-effectiveness of a pharmacogenetic test to guide treatment for major depressive disorder. J Manag Care Spec Pharm. 2018;24(8):726-34. Available at: https://www.jmcp. org/doi/10.18553/jmcp.2018.24.8.726.

13. Phillips KA, Deverka PA, Marshall DA, et al. Methodological issues in assessing the economic value of next-generation sequencing tests: many challenges and not enough solutions. Value Health. 2018;21(9):1033-42.

14. Haga SB, LaPointe NM, Moaddeb J. Challenges to integrating pharmacogenetic testing into medication therapy management. J Manag Care Spec Pharm. 2015;21(4):346-352. Available at: https://www.jmcp.org/ doi/10.18553/jmcp.2015.21.4.346.

15. Watkins JB, Danielson D. Improving the usefulness of budget impact analyses: a U.S. payer perspective. Value Health. 2014;17(1):3-4.

16. Quealy K, Sanger-Katz M. The experts were wrong about the best places for better and cheaper health care. The New York Times. December 15, 2015 Available at: https://www.nytimes.com/interactive/2015/12/15/upshot/thebest-places-for-better-cheaper-health-care-arent-what-experts-thought.html. Accessed June 1, 2019.

17. Yagudina RI, Kulikov AU, Serpik VG, Ugrekhelidze DT. Concept of combining cost-effectiveness analysis and budget impact analysis in health care decision-making. Value Health Reg Issues. 2017;13:61-66.

18. Noridian Healthcare Solutions. Molecular Diagnostic Services (MolDx) Accessed September 23, 2018. Available at: https://med.noridianmedicare. com/web/jeb/policies/moldx. Accessed June 1, 2019.

19. Concert Genetics. Genetic test product catalog. 2018. Available at: https://app.concertgenetics.com/apps/search/\#/. Accessed June 9, 2019.

20. Singh S, Bittner V. Familial hypercholesterolemia—epidemiology, diagnosis, and screening. Curr Atheroscler Rep. 2015;17(2):482.

21. FH Foundation. FIND FH® Heat Map. 2018. Available at: https://thefhfoundation.org/media/FINDFH_heat_map_2.9.2016_V2.pdf?x62576. Accessed June 1, 2019.

22. Husereau D, Drummond M, Petrou S. Consolidated health economic evaluation reporting standards (CHEERS) — explanation and elaboration: a report of the ISPOR Health Economic Evaluations Publication Guidelines Good Reporting Practices Task Force. Value Health. 2013(16):231-50. 